\title{
A Somewhat More Definite Article
}

\author{
Orin Percus \\ University of Massachusetts, Amherst
}

\section{An old challenge}

Take a sentence containing an adverbial quantifier and an indefinite, and often you will be able to paraphrase it with a parallel sentence containing a determiner quantifier and no indefinite. For instance, take the sentences in (1), where the adverbial quantifier is usually/ most of the time and the indefinite is a blue-eyed bear. You can roughly paraphrase these with (2), which contains the determiner quantifier most.

(1) a. A blue-eyed bear is usually intelligent. (von Fintel 1994)

b. Most of the time, a blue-eyed bear is intelligent.

Most blue-eyed bears are intelligent.

This well-known fact suggests that we can use sentences like (1) to express quantification over individuals: to claim that some proportion of individuals (blueeyed bears, say) have some property (intelligence, say). What proportion depends on what adverbial quantifier we use. ${ }^{1}$

Can we explain why these sentences have the use that they do, and at the same time stick to independently motivated syntactic structures and independently motivated rules for interpreting syntactic structures? In this paper, I will take up this old challenge. I will ask why (1) expresses quantification over individuals, and I will give an explanation on which we compositionally interpret structures a lot like those that syntactic evidence points us to. I am addressing the challenge because I want to take a new look at the semantics of indefinites. I will argue that the right explanation involves a new view of the information that the indefinite determiner $a$ contributes. On this view, $a$ carries a uniqueness condition. In particular, I will argue that $a$ has the lexical entry in (3) -- though it is best to ignore the details until Section 3.

The singular indefinite determiner selects for a situation and a predicate of individuals.

$[[a]](\mathrm{s})(\mathrm{P})$ is defined only if there is a unique individual $\mathrm{c}$ in $\mathrm{s}$ such that

$\mathrm{P}(\mathrm{c})=1$. Where defined, its value is that individual.

How might we compositionally derive an interpretation for (1a) that expresses that most blue-eyed bears are intelligent? Here I will briefly sketch one approach to this problem. Aspects of the approach underlie other proposals in the literature (e.g. von Fintel 1994, de Swart 1996) as well as mine.

The surface structure of (1a) is abbreviated in (4). In this structure, usually appears at the VP level and c-commands the small clause complement to be in which the subject is generated. As a first step, let's make the reasonable assumption that the structure we semantically interpret is one where the subject has covertly lowered to its base position, and therefore one that contains the constituents usually and a blue-eyed bear intelligent. Let's imagine moreover that only these constituents are semantically interpreted. ${ }^{2}$ If usually is a quantificational phrase with the force of most, and a blue-eyed bear intelligent is a predicate of 
objects of the kind that usually ranges over, then the combination will tell us that most objects that usually ranges over are objects that a blue-eyed bear intelligent describes.

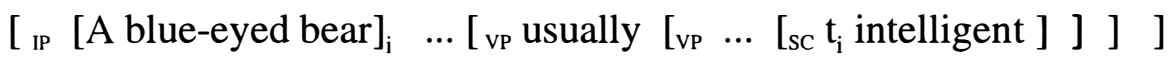

Now, imagine a one-to-one mapping $f$ from some set of objects onto the set of blue-eyed bears. Since $f$ is a one-to-one correspondence, to say that most objects $o$ in the domain of $f$ are such that $f(o)$ is intelligent is just to say that most blue-eyed bears are intelligent. So suppose we can guarantee that usually ranges over all and only the objects in the domain of $f$. Then we are on our way. (1a) will now express that most blue-eyed bears are intelligent as long as our compositional semantics for a blue-eyed bear intelligent insures that, for all $o$ in the domain of $f$, if a blue-eyed bear intelligent holds of $o$, then $f(o)$ is intelligent.

In short, once we take the view that usually combines with a blue-eyed bear intelligent, we can have a compositional analysis as long as we can guarantee two things. One is that usually ranges over the domain of a one-to-one mapping $f$ onto the set of blue-eyed bears. The other is that the constituent a blue-eyed bear intelligent has the kind of semantics that I just outlined. I am going to argue that a denotation for $a$ that includes a uniqueness condition will guarantee both.

\section{The basic outlook}

My proposal here relies on a bigger picture of what we are doing when we use sentences like (1a). In this section I will outline some of the picture. My view is that, to understand why we take a sentence like (1a) to express quantification over individuals, we need to understand some aspects of our pragmatic competence. Specifically, we need to understand this in order to understand how we determine the set of objects that usually ranges over.

Ordinarily, we rely on our pragmatic competence to work out the domain of an adverbial quantifier like usually. ${ }^{3}$ Sometimes, we are guided by clues from the environment, as when I hear (5a) from someone who sees me struggling with the photocopier. Sometimes, we can construct a domain for usually solely on the basis of material in the sentence itself, as we do when we come up with a meaning for $(5 b){ }^{4}$

a. Banging usually helps.

b. That archer usually misses.

Significantly, when we work out the domain of usually, we do so in a reliable way. A speaker counts on this when he uses a sentence like (5a) or (5b): he uses it to mean just what the hearer would take it to mean if the hearer used the operations made available by his pragmatic competence to compute a domain of quantification. My proposal starts from the view that, when we compute the domain of usually in (1a), we use the same machinery that we use in (5a) and (5b). To follow the lines of the sketch, if we take (1a) to express that most blue-eyed bears are intelligent, that must be because our pragmatic competence leads us to a domain in one-to-one correspondence with the set of blue-eyed bears.

At the core of my proposal is this idea: when we evaluate (1a) and conclude that usually's domain is in one-to-one correspondence with the set of blue-eyed 
bears, that is because we are computing usually's domain solely on the basis of sentence-internal information. In other words, we are settling on a domain for usually in the same way that we do when we evaluate $(5 \mathrm{~b})$ in the absence of contextual clues. I will argue that the crucial sentence-internal clue that allows us to determine a domain for usually is a presupposition that the sentence carries, and, more particularly, a uniqueness presupposition that the indefinite determiner gives rise to. On the basis of this clue, our pragmatic strategies apply reliably to secure a domain of quantification.

I can now summarize how, on my proposal, the claim of sentences like (1a) depends on the interpretation of $a$. The interpretation of $a$ enters into the explanation in two different ways. On the one hand, it gives rise to the presupposition that we use to determine the domain of usually. On the other hand, it contributes to the meaning of the constituent that combines with usually -- and in (1a), saying that the meaning of this constituent holds of most items in usually's domain amounts to making a claim about blue-eyed bears. A proposal that follows my basic outlook will have to specify, among other things: an interpretation for $a$; how $a$ 's interpretation contributes to the interpretation of the sister of usually; how $a$ 's interpretation contributes to the presuppositions of the sentence; what the pragmatic strategies are that take us from a sentence's presuppositions to a domain for usually. To the extent that the modes of semantic composition and the pragmatic strategies are independently motivated, we will have a strong argument for the view of the indefinite determiner.

\section{Preliminaries}

In presenting my analysis, I will be making a number of technical assumptions. Here is a very quick summary.

First, I will assume that the representation that serves as input to semantic interpretation is a logical form (lf) roughly in the style of Heim and Kratzer 1998. These lfs are trees that differ slightly from the output of the initial syntactic component of the grammar. One way in which they differ is that they contain additional nodes. Some of these nodes are occupied by objects that are no different from pronouns as far as their interpretation goes. Others are occupied by operators: these operators are coindexed with items that occupy other positions, and they function as variable abstractors. We will see examples shortly. Each constituent $X$ in the tree has a denotation $[[X]]$, and the denotation of a mother node is obtained from its daughters' denotations by rules of functional application or predicate abstraction.

To make things easier here, I am going to simplify the lfs of the sentences we are concerned with, and I am also going to make simplifying assumptions about the denotations of their constituents. Where lfs are concerned, I will omit nodes when they are not immediately relevant for my purposes, including those nodes that are reserved for tense morphemes. ${ }^{5}$ As for denotations, I will assume that sentences have denotations that are truth values and that they are constrained to "talk about the actual world." That is, a language user will take all expressions of basic types (more precisely, all such expressions that do not appear within the clausal argument of a modal or attitude verb) to denote objects in his construction of the actual world; he will take all predicates (with the same qualifications) to hold only of objects in his construction of the actual world; he will judge a sentence with logical form $L$ to be true as long as on his construction of the actual world $[[\mathrm{L}]]=$ 1. 
An important semantic assumption of mine is that adverbial quantifiers like usually range over situations in roughly the sense of Kratzer 1989, where situations are parts of worlds. (I won't be very precise in talking about these objects: I will speak of some situations as containing individuals, but I won't clarify exactly what this means, and I hope that the discussion will seem coherent. ${ }^{6}$ ) Adverbial quantifiers relate two predicates of situations, so that the lexical entry of usually specifies the following:

Where defined, [[usually] $](\mathrm{p})(\mathrm{q})=1$ iff most situations $s$ such that $\mathrm{p}(s)=1$ are such that $\mathrm{q}(s)=1$.

Accordingly, usually combines with two items at logical form, each of which is interpreted as a predicate of situations. Basically following von Fintel 1994, I will assume that the item that functions as the quantifier's restrictive argument -- an item that is possibly not present in the initial syntactic output and is at any rate unpronounced - is interpreted in just the way a pronoun is. It's a "propredicate." A hearer, in order to interpret a sentence with usually, must locate an antecedent for this propredicate ${ }^{7}$ - that is, an object that can serve as the propredicate's value. On this way of looking at things, the problem of finding a domain of quantification for usually is the problem of finding an antecedent for the propredicate. In general, what antecedents are available? I will assume for one thing that, when the context makes salient a set of situations, the characteristic function of this set is a possible antecedent. If a hearer imagines that no contextually salient set of situations provides an antecedent, then to find an antecedent he must use strategies that his pragmatic competence makes available.

\section{The solution}

I will now show how the lexical entry for $a$ in (7) $(=(3))$ leads us to the impression that $(8)(=(1 \mathrm{a}))$ claims that most blue-eyed bears are intelligent.

(7) The singular indefinite determiner selects for a situation and a predicate of individuals. $[[\mathrm{a}]](\mathrm{s})(\mathrm{P})$ is defined only if there is a unique individual $\mathrm{c}$ in $\mathrm{s}$ such that $\mathrm{P}(\mathrm{c})=1$. Where defined, its value is that individual.

A blue-eyed bear is usually intelligent.

The (simplified) logical form for (8) is as in (9).

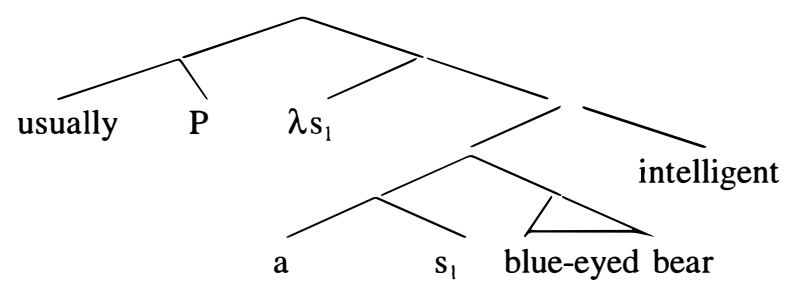

Note that the $\lambda s_{1}$ operator binds the position occupied by $a$ 's situation argument, thereby creating the predicate of situations that serves as usually's second argument. The first argument of usually -- its sister, $P$-- is a "propredicate" whose antecedent we must find if we want to interpret the sentence. I won't go into the details, but on plausible assumptions about the denotations of lexical items, our 
rules of semantic composition will predict that we judge (8) to be true as long as (on our construction of the world) most situations s such that $[[P]](s)=1$ are such that the unique blue-eyed bear in $s$ is intelligent. (In what follows, I will call the situations of which [[P]] holds P-situations. I will also abbreviate blue-eyed bear as beb.)

Now recall the idea. When a hearer has to interpret the sentence and understands that no contextually salient set of situations provides an antecedent for $\mathrm{P}$, he will reliably assume that the set of $\mathrm{P}$-situations is in one-to-one correspondence with the set of all blue-eyed bears. Here is how this happens.

\subsection{The starting point}

The starting point is this: the indefinite determiner in (8) gives rise to a presupposition ${ }^{8}$ that every $P$-situation contains a single beb.

How come? The presupposition here is a presupposition that a certain property (the property of containing a blue-eyed bear) holds of every item in the domain of usually. In other quantificational sentences, we also find the presupposition that a certain property holds of every item in the quantifier's domain. For instance, generally it is proper to utter the sentence in (10a) only if it is established that all of the people under discussion hid in the living room. ${ }^{9}$ I claim that the presupposition here arises in the same way that it does in those other sentences.

a. Exactly half of them regretted hiding in the living room.

b. [Exactly half of them] $\left[\lambda \mathrm{x}_{2} \quad\left[{ }_{\beta} \mathrm{t}_{2}\right.\right.$ regretted hiding in the living room ]]

To understand the source of the presupposition in (9), let's start by considering the presupposition in (10a). Why is the presupposition of (10a) that all of the individuals in the domain of exactly half hid in the living room? This is related to the fact that a simple sentence like (11) presupposes that Vladimir hid in the living room.

Vladimir regretted hiding in the living room.

Informally, sentences like (10a) obey the following pattern: Suppose a sentence that ascribes property $Z$ to some entity presupposes that the entity has another property $Z^{*}$. Then take a new sentence that says that some proportion of entities with property $Y$ have property $Z$; the new sentence presupposes that every entity with property $Y$ has property $Z^{*} .^{10}$ To be a little more precise about what is going on, we want to talk about the logical form of sentences like (10a) and how pieces of logical form compositionally affect the presuppositions of the sentence. What I am claiming is roughly this. In (10a) as in (11), the VP introduces a requirement that has to do with hiding in the living room. In (10a), combining the quantificational phrase with the VP makes this requirement hold for every individual in the quantifier's domain. In exact analogy, the VP in (9) introduces a requirement that has to do with containing a single beb, and combining usually with the VP makes this requirement hold for every situation in the quantifier's domain.

To discuss the requirements that pieces of logical form introduce, I will use the technical term PRESUPPOSITION (in capitals). Let's say that pieces of logical form PRESUPPOSE, that in particular truth-value denoting constituents PRESUPPOSE claims, and that a sentence presupposes that $\mathrm{p}$ as long as its logical form PRESUPPOSES that p. ${ }^{11}$ Then what I just said translates as follows. (11)'s If 
PRESUPPOSES that Vladimir hid in the living room. In just the same way, we can argue, the constituent $\beta$ in (10a)'s lf (abbreviated in (10b)) PRESUPPOSES that $\left[\left[\mathrm{t}_{2}\right]\right]$ hid in the living room. Our observation about the presuppositions of (10a) shows that, on the basis of this, the rules we have for determining PRESUPPOSITIONS yield the following PRESUPPOSITION for the full lf in (10b): every individual in exactly half's domain hid in the living room. I claim that, in exact analogy, the lf constituent [ $a s$, blue-eyed bear intelligent] PRESUPPOSES that $\left[\left[\mathrm{s}_{1}\right]\right]$ contains a single beb, and the full lf in (9) PRESUPPOSES that every situation in the domain of usually contains a single beb.

Why does the lf constituent $a s_{1}$ blue-eyed bear intelligent PRESUPPOSE that $\left[\left[\mathrm{s}_{1}\right]\right]$ contains a single beb? Because of the uniqueness condition in the lexical entry for $a$. Since $[[a]]\left(\left[\left[\mathrm{s}_{1}\right]\right]\right)([[\mathrm{beb}]])$ is defined only if $\left[\left[\mathrm{s}_{1}\right]\right]$ contains a single beb, our semantic composition rules make [[a $s_{1}$ beb intelligent]] defined only if $\left[\left[s_{1}\right]\right]$ contains a single beb. I will adopt the widespread view that definedness conditions give rise to felicity conditions ${ }^{12}$, and assume specifically that a logical form PRESUPPOSES that its denotation is defined. Since [ $\left[a s_{1}\right.$ beb intelligent $\left.]\right]$ is defined only if $\left[\left[\mathrm{s}_{1}\right]\right]$ contains a single beb, that means the lf constituent $a s_{1}$ beb intelligent PRESUPPOSES that $\left[\left[\mathrm{s}_{1}\right]\right]$ contains a single beb.

To summarize, the presupposition that every P-situation contains a single beb arises from three factors: the uniqueness condition in the lexical entry for $a$ together with the compositional rules for determining denotations; the way in which definedness conditions on denotations give rise to presuppositions; and the compositional way in which presuppositions are determined. At the bottom, crucially, is the uniqueness condition of $a$.

\subsection{The search for an antecedent}

Once the hearer calculates the presupposition that every P-situation contains a single blue-eyed bear, pragmatic strategies will lead him to a value for P. Here is how.

The first strategy that the hearer uses is to assume that the presupposition of (8) is true -- in other words, to act as though, at least as far as presuppositions go, the speaker spoke appropriately. So the hearer concludes that every P-situation contains a single blue-eyed bear.

The next strategy that the hearer uses, I claim, involves a default assumption. Informally, the strategy says: if you know of some relation that it pairs each member of one set with a unique member of another, then assume it pairs each member of the first set with a different member of the second, unless you have good reason to believe otherwise. In this case, the hearer believes that the contain-relation pairs each P-situation with a single beb, and so he assumes that no two P-situations contain the same beb. One way of looking at this step is that, as part of our attempt to narrow down the identity of $\mathrm{P}$, we narrow down the identity of a certain function from situations to bebs, a function whose domain is the set of P-situations. We do so by assuming the function is one-to-one. (Accordingly, it might be right to think of the strategy as following from a more general default that takes functions to be one-to-one.)

The final step that the hearer takes is to choose a value for $\mathrm{P}$ such that $\mathrm{P}$ situations partition the world of evaluation. If P-situations partition the world of evaluation, then every beb is contained in some P-situation. And since every Psituation contains a different beb, this guarantees that there are exactly as many Psituations as bebs. We now have a one-to-one mapping from $\mathrm{P}$-situations onto the set of blue-eyed bears, a mapping that pairs each P-situation with the blue-eyed bear it contains. 


\subsection{The result}

The solution is now in place. Pragmatic strategies have chosen a value for $\mathrm{P}$ such that there is a one-to-one mapping $f$ from P-situations onto blue-eyed bears that pairs each P-situation with the blue-eyed bear it contains. The rules of semantic composition tell us that (8) is true as long as most P-situations $s$ are such that the unique blue-eyed bear in $s$ is intelligent, or in other words as long as most Psituations $s$ are such that $f(s)$ is intelligent. Or, since $f$ is a one-to-one mapping from P-situations onto blue-eyed bears: as long as most blue-eyed bears are intelligent.

The stronger the motivation for the pragmatic strategies I cited, the stronger the argument that $a$ has the meaning I proposed. I will just note here that there is some evidence that we use these strategies. (See Percus 1997 for more thorough discussion.) As far as the first strategy is concerned, I think it is uncontroversial that, when the context supplies no possible antecedent for an anaphoric expression, we can make use of the presuppositions of the sentence to come up with one. ${ }^{13}$ As for the second default strategy -- the one that leads us to conclude that each Psituation contains a different beb once we know that each P-situation contains a single beb -- we seem to use it in cases like the following. I think it is natural to judge (12b) to be an entailment of (12a), although strictly speaking this is not correct. It is correct if every student occupies a different apartment. It is plausible that we make this judgment because, when evaluating (12a) out of context, we make use of the same strategies that we make use of in (8). (12a) carries a presupposition with universal force: it presupposes that each student in the domain of quantification occupies a single apartment. But once we calculate this, we apply the second default strategy and assume that each student occupies a different apartment. $^{14}$

(12) a. Most students keep their apartment clean.

b. Most student apartments are clean.

\section{Why not quantification over individuals?}

I set out to explain why sentences like $(13)(=(1 \mathrm{a}) /(8))$ appear to quantify over individuals. I gave an analysis under which the semantics of sentences like (13) involves quantification over situations. An alternative is to endorse a theory under which these sentences directly quantify over individuals -- under which (13), for instance, directly quantifies over blue-eyed bears, and has a semantics just like the determiner quantifier paraphrase in (14). In fact, one might take the view that the simplest way of explaining why (13) and (14) appear to express the same thing is to say that their semantics is identical.

(13) A blue-eyed bear is usually intelligent.

(14) Most blue-eyed bears are intelligent.

Now, it isn't hard to criticize theories that say that sentences with adverbial quantifers and parallel sentences with determiner quantifiers express the same thing because they have identical logical forms. This position (which one finds in some variants of the theories developed by Heim 1982 and Kamp 1981) leads to a number of problems, which I will just mention quickly. It wrongly predicts that the two kinds of sentences should contribute in just the same way to sentences in which 
they are embedded. They don't: the antecedent in (15a) talks about a possible state of affairs, but the antecedent in (15b) talks about an impossible one. ${ }^{15}$

a. If every semanticist was a syntactician instead, a lot more would get done in our field.

b. ?? If a semanticist was always a syntactician instead, a lot more would get done in our field.

It also predicts that the two should behave the same way with respect to wellformedness conditions on logical forms. This is not so clear. The near minimal pair of sentences in (16) should get essentially the same logical form (the same but for the identity of the quantifier, which has different force in the two examples). Assuming that what rules out one reading of (16a) is a condition on logical forms, we should then expect the analogous reading of (16b) to be absent, but it's there.

a. \# Only his office mate likes every student.

b. Usually, only his office mate likes a student.

But can we argue against the more basic idea that the semantics of sentences like (13) involves direct quantification over individuals?

In what follows, I will consider a sentence more complex than (13), one that like (13) contains an adverbial quantifier and an indefinite. I think that, just as with (13), we are tempted to imagine that this sentence involves quantification over individuals. However, I will argue that this approach to it is wrong. If you think that a theory that predicts that (13) involves quantification over individuals will also predict that this other sentence admits an interpretation involving quantification over individuals, then you can take what I say as an argument against such a theory. If you think not, then what I have to say simply serves as a warning with regard to sentences like (13).

My tactic here is to consider a case where, in evaluating a sentence with an adverbial quantifier and an indefinite, we clearly make use of contextual information. In particular, I will examine a sentence that can express quantification over a contextually restricted set of individuals. That sentence is (17). The point is this: we only take the sentence to express quantification over a contextually restricted set of individuals when there is a contextually salient set of situations that the individuals are in a one-to-one correspondence with.

(17) Ursula usually knew whether a blue-eyed bear was intelligent.

Here first is something that might persuade us that the semantics of (17) involves quantification over individuals. Imagine the following scenario:

Background. Ursula has agreed to be a subject in an experiment, and she has the following task to perform. She watches as blue-eyed bears walk out from behind a screen and then walk behind it again, one at a time, and her task is to say whether the bear that just walked out is intelligent or not.

Scenario One. In Scenario One, a different beb walks out each time. For most of those bears, it is quite clear to Ursula whether or not they are intelligent. For concreteness: there are 50 bears total; for 40 of those bears, it is absolutely clear to Ursula (for whatever reason) whether they are intelligent or not; for 10 of them she hasn't the faintest idea (and says so). So 10 out of 50 times that Ursula is confronted with a beb, she has no idea whether the bear she is confronted with is intelligent, and 40 out of 50 times that she is 
confronted with a beb, she knows whether the bear that she is confronted with is intelligent.

If we evaluate (17) against the background of this scenario, we think that it truly reports the results of the experiment. We might conclude that the semantics of (17) states that most of the bebs in the contextually salient set of individuals are such that Ursula knew whether they were intelligent. The contextually salient set of individuals here is the set containing Ursula and the bebs she is confronted with. There is independent evidence that this set can be used to restrict the domain of quantifiers. When we evaluate (18), for example, we naturally interpret it as making the claim that most of the bebs in the experiment did not understand the purpose of the experiment.

Most blue-eyed bears did not understand the purpose of the experiment.

But (17) does not always express quantification over individuals in the contextually salient set. Consider Scenario Two:

Scenario Two. In Scenario Two, sometimes the same beb walks out more than once. It is still true that most of the bears that Ursula is confronted with are transparently intelligent or unintelligent. But what is remarkable about this scenario is that, as it happens, Ursula is confronted most of the time with bebs whose intelligence she is unsure of. They just walk out much more often than the other bears do. For concreteness: 10 bears total; for 8 of those 10 bears, it is absolutely clear to Ursula whether they are intelligent or not; for 2 of the 10, she hasn't the faintest idea. But those two bears of unfathomable intelligence walk out from behind the screen a total of 40 out of 48 times, and all the others one time each. So, 40 out of 48 times that Ursula is confronted with a beb, she has no idea whether the bear she is confronted with is intelligent, and, 8 out of 48 times that she is confronted with a beb, she knows whether the bear that she is confronted with is intelligent.

Here, the intuition is that (17) does not adequately report the experimental results. But it is true that most of the bebs in the contextually salient set are such that Ursula knew whether they were intelligent. This is unexpected if the semantics of (17) is as we conjectured.

What is the difference between the two scenarios? In the first, each trial involves a different bear. In the second, there are trials that involve the same bear. In other words, (17) seems to express that Ursula correctly assessed the intelligence of most contextually salient blue-eyed bears only when it so happens that in most trials Ursula correctly assessed the intelligence of the bear she was looking at. Now, we have independent reason for believing that usually can range over the set of trials. We naturally understand (19) as saying that in most trials -- or on most occasions when Ursula was confronted with a beb -- Ursula took under thirty seconds to respond. The pattern of judgments that we find suggests that, even when (17) seems to express quantification over bebs, it is actually quantifying over trials.

Ursula usually took thirty seconds to respond.

I take it that these scenarios make salient a set of situations, each of which corresponds to a trial in the experiment. If that is right, we can summarize as follows. We considered a single sentence containing an adverbial quantifier and an indefinite, and we looked at it against two background scenarios. Each scenario 
made salient a set of situations and a set of individuals. We saw that the sentence could express quantification over individuals in the contextually salient set when they were in one-to-one correspondence with the situations, but not when they were not. This is mysterious if the semantics of the sentence involves explicit quantification over individuals: what makes the set of salient bebs a possible domain of quantification in one case but not the other? However, it is unsurprising if the semantics of the sentence involves quantification over situations, and a set of salient situations can generally serve as the domain of quantification.

Here, for instance, is what my proposal would say about these cases. I will try to sidestep issues in the syntax and semantics of interrogatives by assuming a simplified lf like (20), where know whether is an unanalyzed lexical item with a semantics roughly like this: [[know-whether]] $(s)(p)($ Ursula $)=1$ as long as either (i) $p$ holds of $s$ and, in $s$, Ursula "believes of" $s$ that $p$ holds of it, or (ii) $p$ does not hold of $s$ and, in $s$, Ursula "believes of" $s$ that $p$ fails to hold of it. ${ }^{16}$

$$
\text { usually } \left.P\left[\lambda \mathrm{s}_{1} \text { Ursula [[know-whether } \mathrm{s}_{1}\right]\left[\lambda \mathrm{s}_{2} \text { a } \mathrm{s}_{2} \text { beb intelligent }\right]\right]
$$

In brief, the rules of semantic composition will tell us that $[[(20)]]=1$ as long as most P-situations s are such that Ursula knows in $s$ whether the unique beb in $s$ is intelligent. Moreover, presuppositions will "project" in such a way as to insure that every P-situation contains (Ursula and) a single blue-eyed bear. (See note 16 for the definedness condition of know-whether, which plays a role in generating this presupposition.) Both scenarios make salient a set of situations of this kind -- the set of trial situations - and accordingly both make available a possible antecedcent for $P$. If our preference is to use this antecedent, then we can explain our judgments. In the first scenario, it is true that, for most trial situations $s$, Ursula knew in $s$ whether the unique bear in $s$ was intelligent, so we will judge (17) to be true. In the second, it isn't, so we won't. ${ }^{17}$

In short, when we look at cases where adverbial quantifiers and indefinites express quantification over a contextually restricted set of individuals, we can show that their semantics involves explicit quantification over situations, not individuals. This suggests that the same holds for cases where such sentences express quantification over a set of individuals without contextual restriction.

\section{An alternative}

As my opening sketch foreshadowed, my account has the following features: the adverb in sentences like (13) quantifies over something situation-like; and the constituent that functions as the adverb's scope argument describes a situation $s$ in the adverb's domain only if $f(s)$ is intelligent, $f$ a one-to-one mapping from situations in the adverb's domain onto blue-eyed bears. I am not alone in proposing an account with these features: some other recent proposals with the same shape are von Fintel's $(1994,1996)$ and de Swart's (1996). ${ }^{18}$ At the same time, these researchers make use of different building materials, and in particular they don't assume that indefinites come with a uniqueness requirement. Here I want to argue against von Fintel's proposal, which is the one I understand best. In doing so, I will present what I take to be the basic structure of von Fintel's analysis, departing from details in ways that I deem appropriate.

Von Fintel's account is like mine conceptually (and largely inspired mine). At logical form, usually combines with a "propredicate" that describes situations (I will call it $P$ again); we imagine that the context furnishes no antecedent for this 
predicate when we evaluate (13), and we use pragmatic strategies to lead us to an antecedent; we are guided to an antecedent by a presupposition that (13) carries. But for von Fintel the truth conditions of (13) are different and, moreover, the presupposition that (13) carries is weaker: (13) presupposes that every P-situation contains at least one blue-eyed bear. ${ }^{19}$ The weaker presuppositions that von Fintel's theory predicts are, I will argue, its downfall.

Von Fintel follows a program with two strands. One is that the indefinite determiner is a quantifier with existential force; the other is that natural language predicates express persistent properties of situations. The first strand leads to the following denotation for the scope argument of usually in (13): [ $\lambda s$. $s$ contains at least one intelligent blue-eyed bear]. The second suggests a lexical entry for usually that looks at the predicate of situations that it combines with first, takes its characteristic set, and chooses for its domain only those elements in the set that have no proper parts in the set. Specifically: where defined, [[usually]] $(p)(q)=1$ iff most situations $s^{\prime}$ such that $s^{\prime} \in \operatorname{MIN}(\{\mathrm{s}: \mathrm{p}(\mathrm{s})=1\})$ are part of some situation $s^{\prime \prime}$, where $s^{\prime \prime} \in \operatorname{MIN}(\{\mathrm{s}: \mathrm{p}(\mathrm{s})=1 \& \mathrm{q}(\mathrm{s})=1\})$. (Or to put it another way: ...iff most minimal situations that make its first argument true are part of a minimal situation that makes its second argument true. In the formulation here, $\operatorname{MIN}(\mathrm{S})=$ $\{\mathrm{s}: \mathrm{s}$ is in $\mathrm{S}$ and no proper part of $\mathrm{s}$ is in $\mathrm{S}\}$. ) Together, these insure that $[[(13)]]^{20}$ $=1$ only if most minimal elements of the set of $\mathrm{P}$-situations are part of a minimal element of the set of P-situations containing at least one beb. What will happen when there is no antecedent for P? We know that every P-situation contains at least one beb. Von Fintel (as I represent him) assumes that our pragmatic competence will lead us to the set that contains all parts of the evaluation world that contain at least one beb. Now, the minimal elements of this set are situations that contain a single beb (and nothing else), and, crucially, each such situation will contain a different beb. This means that, in ranging over minimal elements of this set, usually will range over situations that are in one-to-one correspondence with bebs, just as on my account. [[(13)]] will then be 1 as long as most of these situations are part of a situation containing a single intelligent beb (and nothing else). But a situation containing a single beb and nothing else is part of a situation containing a single intelligent beb and nothing else only if the beb in the first situation is intelligent. So [[(13)]] will be 1 as long as most bebs are intelligent.

The important thing to note about this account is that it has a very different way of determining that usually ranges over situations that contain a single beb. The sentence's weak presupposition admits $\mathrm{P}$-situations that contain more than one beb, but the picky quantifier eliminates them from its domain when they have proper parts that are also P-situations. The weak presupposition means, among other things, that a set of situations that cannot provide an antecedent for $\mathrm{P}$ on my account can provide an antecedent for P on von Fintel's. Accordingly, we might expect that, when we evaluate sentences like (13) in context, von Fintel will predict that the sentences can make claims that my analysis predicts they can't make. I will argue now that cases where we evaluate sentences like (13) in context provide evidence for my analysis over von Fintel's.

We have seen that the context can play a role in determining the claim that (21) $(=(17))$ makes, so let's consider (21) again.

(21) Ursula usually knew whether a blue-eyed bear was intelligent.

Von Fintel's analysis (as I cast it) says this about (21). The denotation of (21) is 1 as long as most minimal P-situations are part of a minimal element of the following 
set: the set of all P-situations $s$ such that Ursula knows in $s$ whether $s$ contains at least one intelligent beb. The presupposition of (21) is the same as that of (13): every P-situation contains at least one beb. (I will ask you to take my word for this, since I haven't explained how these presuppositions are derived on von Fintel's analysis.)

Now here is a scenario that differs slightly from the two we considered earlier.

Scenario Three. This experiment is slightly different. Now Ursula is confronted with four blue-eyed bears at a time, though her actual task is still to say for each of those bears whether the bear is intelligent.

Now, as everyone knows, getting a group of blue-eyed bears together can mean trouble. If none of them are very bright, they immediately get into a fight. Still, if there does happen to be an intelligent blue-eyed bear among them, the intelligent one generally gets the others to curb their disorderly tendencies, and manages to keep the group tolerably well behaved. What this means, of course, is that, when you are confronted with a group of blue-eyed bears, it is pretty easy to tell whether at least one of them is intelligent. If the entire group is well behaved, then there has got to be an intelligent bear among them. On the other hand, if they are embarrassingly unruly, then it's a pretty good bet that none of them is intelligent.

What actually happens is this. Ursula sees ten groups of bears. Three are riotous; the others are well behaved. As far as the riotous groups go, Ursula immediately responds that not a single bear in the group is intelligent. She is correct for the most part: actually, one of the groups contains a bear that is intelligent but not such a great disciplinarian. As far as the intelligent groups go, Ursula is at a loss. She is able to tell that at least one bear in the group is intelligent, and she tells the experimenters this at once. But she has no clue which bears are the intelligent ones, and when pressed to respond about each bear in the group, her responses are random.

What does von Fintel predict our judgment to be when we evaluate (21) with this scenario in mind?

My answer to this question depends on two assumptions. The first is that von Fintel will say that the context makes salient a set of situations whose minimal elements are trials in the experiment. (Why assume this? Well, there is independent evidence that usually can range over the set of trials -- we take a sentence like Ursula usually didn't want to give a response to mean that, in most trials, Ursula didn't want to give a response. So it is natural to think that a set is salient whose minimal elements are trials, and that we use this set to obtain an antecedent for the propredicate that is usually's first argument.) The second is that in evaluating (21) we can use this set to obtain an antecedent for $\mathrm{P} .{ }^{21}$ Note that, if the minimal P-situations are trial situations, the presupposition of (21) will not be violated, because each trial situation (as well as every situation containing it) contains at least one beb -- it just so happens that each trial situation contains more than one beb. Now, if the minimal P-situations are trial situations, we should judge (21) to be true: in Scenario Three, most individual trial situations are (part of) individual trial situations where Ursula knows whether at least one beb is intelligent. But this prediction is wrong: we definitely don't judge (21) to be true.

What does my analysis predict? If as on Scenario One and Scenario Two we take the set of P-situations to be the set of trials, then (21)'s presupposition will not be satisfied, and (21) will just seem inappropriate. If we don't, our default strategies will guide us towards P-situations that are not restricted to the scenario at 
hand. Either way, (21) will seem bizarre as a claim about the scenario. I think this is right.

\section{The odds, and loose ends}

Can the proposal here serve as a starting point for another view of the semantics of sentences with indefinites? Here are some potentially good signs that require serious investigation. First, there are other cases where the indefinite determiner seems to come along with a uniqueness requirement. I have in mind here "specificational" sentences with indefinite subjects, which behave as though their subjects denote an individual:

A book that might be of use to you is Autumn in Peking. \# A book that might be of use to you is also Heartsnatcher.

Second, the analysis of $a$ that I have given is a plausible candidate for the $a$ of "specific indefinites" (given that the situation argument of $a$ can be interpreted referentially or bound non-locally, say by an existential quantifier over situations).

At the same time, even the small amount that I presented here involved many simplifications. Throughout this discussion, I made use of ultrastreamlined logical forms and accordingly simplified denotations. More sophisticated lfs might include, among other things, a situation abstractor at the level of the root node (if sentences are functions from situations to truth values) and existential quantifiers over situations (quantificational analyses of tense, e.g Ogihara 1996, Heim 1997, motivate two: one for the tense morpheme itself, with scope above determiner quantifiers, and a covert one, with scope below). It is important to consider what more sophisticated lfs might look like, for at least two reasons. First, we want to verify that assumptions of the kind I made here transfer naturally to accounts with more complicated lfs and meanings. Second, complicating the lfs and denotations might give us clues to what seem now to be forbidding puzzles. One obvious puzzle is: what should be said about those cases of indefinites that convinced everyone from Russell onwards that indefinites were existential quantifiers? While it's conceivable that the determiner in these cases is just a different lexical item, it could also be that a covert existential quantifier over situations is binding the determiner's situation argument. Another puzzle is: what should be said about examples like (23) that pose what Heim 1990 in a similar context called the "indistinguishable participant" problem?

A bishop usually respects a clergyman from his own country.

(Glossing over some details,) if both indefinites in (23) contain a situation position bound by usually, then on an analysis like mine (23) will end up presupposing that every P-situation contains a unique bishop and a unique clergyman from the bishop's country. But this means that every P-situation has to contain a unique bishop and no other clergyman from the bishop's country, and so (23) should express, if anything, something like what is expressed by A bishop usually respects himself. Wrong. Just to begin to get out of this problem, we will have to say that, in the actual if for (23), the two situation arguments do not have the same value. We might therefore suppose that (23) contains at least two quantifiers over situations (relevant here are the covert quasi-universal quantifiers posited elsewhere) and that each one of the two quantifiers binds one situation argument. Of course, this is only the first step. Just for the record, by the way, some 
sentences like (23) do not make coherent claims -- $(24 a, b)$ for example -- and it looks reasonable to attribute this to the fact that two situation arguments are receiving the same value. This suggests to me that the "indistinguishable participant" problem does not pose a deep problem for my approach.

a. ?? A bishop and another bishop usually respect each other.

b. ?? A bishop and a clergyman from his country usually respect each other.

(cf. c. A bishop and a cardinal usually respect each other.)

I will conclude with the most important, and most obvious, loose end. I have proposed a semantics for the indefinite determiner that is suspiciously like the semantics that one might expect for the definite determiner. But of course indefinites and definites are not identical in their use. What is the difference between the two? I blushingly admit that I don't know. However, my hope is that we can say that the main difference between $a$ and the has to do with their selectional requirements -- that $a$ takes a situation argument while the does not -and that the differences in use in some manner follow from this. Suppose that the simply selects for a predicate of individuals and yields the unique individual that satisfies the predicate. We might then be able to get a handle on why, unlike (25a), (25b) does not seem appropriate to describe a generalization about barbers. For (25b) to describe a generalization about barbers, the predicate that the combines with at logical form must somewhere inside of it contain a situation position bound by usually. If the denotation of barber is the same in (25a) and (25b) and does not contain such a position, then this means that we must find some way of providing such a position at logical form (maybe by combining barber with some silent constituent that includes a situation position). Perhaps it is difficult to do so. ${ }^{22}$

a. A barber is usually bald.

b. \# The barber is usually bald.

How about cases where we can use definites but not indefinites, (26) for example?
a. ?? A brightest star is usually Vega.
b. The brightest star is usually Vega.

Without going into detail, I hope that we can put at least some of these cases down to a preference for choosing logical forms with the in cases where the nominal predicate is known to hold of only one individual. Needless to say, all this is quite speculative. I would like you to think of this ending as suspenseful.

\section{Endnotes}

*This paper reports results of my thesis (Percus 1997), and some of the issues here are dealt with in greater detail there. The work reported here obviously owes a great deal to the work of Kai von Fintel and Irene Heim, but over and above that it owes a lot to their encouragement and support. Thanks to Danny Fox and Jon Nissenbaum for some last minute comments.

1. (1) is said to exemplify the "quantificational variability" of indefinites. The idea is that, if you imagine that in sentences like (1) the indefinite magically transforms into a DP with a quantificational determiner, the quantificational force of this determiner will vary according to the quantificational force of the adverbial. 
2. Throughout this paper, I will assume that be is not interpreted and I will therefore ignore its presence in the input to interpretation.

3. By the domain of a quantifier I mean the set of objects it ranges over.

4. See Schubert and Pelletier 1987 for some famous examples. Other classic cases where we construct a quantifier domain purely on the basis of sentential material are cases where a quantifier associates with focus, if Rooth 1992, von Fintel 1994, Buering 1996 and others are on the right track.

5. One simplification to note is this. Although I assume that the main predicate of every clause selects for a situation argument and combines at logical form with a situation "pronoun," I will sometimes leave out these situation "pronouns" when for my purposes here their semantic contribution is negligible. For instance, I will leave out the situation argument of intelligent from my logical forms. (What makes this possible is that, if $s_{1}$ and $s_{2}$ are in the same world, then the functions [[intelligent]] $\left(s_{I}\right)$ and [[intelligent]] $\left(s_{2}\right)$ are suitably alike -- they tend to yield the same truth value for any individual in the domain of both.)

6 . I won't clarify the way I use the word "individual" here, but I will note that, for me, "situation" and "individual" talk about different ontological categories. A situation may contain an individual and in fact nothing other than an individual, but that does not make an individual a situation.

7. Unless the propredicate is coindexed with an operator. Then it functions as a bound variable.

8. I use the term "presupposition" here in a way that connects to pragmatics: basically, when a sentence presupposes that $\mathrm{p}$, a speaker can use the sentence only if the interlocutors have agreed to take $\mathrm{p}$ for granted. Though in the text I treat this notion as important, this is just for convenience in presentation. The notion that I take to be more basic is the notion of PRESUPPOSITION below, and the way in which the rules of conversation regulate a sentence's use is in part mediated by this notion -- the rules of conversation dictate that a speaker can use a sentence with a logical form L only if the interlocutors have agreed to take the PRESUPPOSITION of L for granted.

9. The example is inspired by one from Beaver 1995. Here is evidence for my claim. Imagine that we are talking about a game of hide-and-seek in which 20 boys were playing, and I know that 16 of the 20 hid in the living room, that 10 of those 16 regretted hiding there, and also that 10 of those 16 hid behind the grand piano. I could then truthfully report (i a) -- indicating that I can in principle use them to refer to the entire group of boys that most ranges over. But I could not truthfully report (i b) -- indicating that in (i b) I cannot use them to refer to this group. Why can't I? Because the second clause of (i b) presupposes that every individual in the domain of exactly half hid in the living room.

(i) a. Most of the boys hid in the living room, and exactly half of them hid behind the grand piano.

b. Most of the boys hid in the living room, and exactly half of them regretted hiding there.

10. Heim made use of this generalization in formulating her 1983 theory of presupposition projection. Not all quantificational sentences conform to it (see in particular Beaver 1995 for discussion of this point). This is a potential worry, but it does seem to me that the exceptions fall into categories that (8) does not belong to (I unfortunately don't have the space to justify this here).

11. The precise way I am presenting this depends on my simplified assumptions about sentence denotations; other views could yield the same basic result.

12. Soames 1989 criticizes this idea. It isn't crucial to my general account. If definedness conditions don't give rise to PRESUPPOSITIONS, we can adopt a 
minimally different theory where we replace the definedness condition in the lexical entry of $a$ with whatever will compositionally give rise to the PRESUPP. we need.

13. An important further observation that emerges from the discussion of intermediate accommodation in Beaver 1995 and von Fintel 1996 is that, when the context does make available a possible antecedent, we often prefer using it to constructing one ourselves.

14. Is there motivation for the final step, where the hearer assumes that P-situations partition the world of evaluation? Answering this question requires fleshing out the idea more. Recall that we can resolve $\mathrm{P}$ by using the characteristic function of a salient set of situations. Maybe in this case, where we choose not to make use of any contextually salient set of situations, we "generate" a set of situations that we can make use of. Perhaps one could motivate the idea that one option that we have for "generating" a set of objects is to partition an already salient object, and that the world of evaluation is always salient.

15. I discuss this phenomenon in Percus 1998.

16. When I say that $p$ holds of $s$ here, I mean that $p(s)=1$. Here is a sloppy way of spelling out in a possible worlds semantics that, in $s$, Ursula "believes of" $s$ that $p$ holds of it: in all worlds $w$ compatible with Ursula's thoughts in $s, p(\Sigma(w))=1$, where Ursula represents $s$ to herself via the concept $\Sigma$. So to be a little more precise about the semantics: where defined, [[know-whether]] $(s)(p)(x)=1$ iff either (i) $p(s)=1$ and, for all worlds $w$ compatible with $x$ 's thoughts in $s, p(\Sigma(w))$ $=1$, or (ii) $p(s)=0$ and, for all worlds $w$ compatible..., $p(\Sigma(w))=0$ ( $\Sigma$ being the concept via which $x$ represents $s$ to himself). I will assume here that [[know whether $]](s)(p)(x)$ is defined only if $x$ is in $s$ and $p(s)$ is defined. (The intuitive motivation for this is that sentences of the form $A$ knows whether $B$ generally presuppose what $B$ on its own presupposes, but I have not thought in detail about the facts. We might want a slightly different definedness condition.)

17. Note in passing that we might have some evidence here that in sentences like our old friend $(1 \mathrm{a}) /(8) /(13)$ a pragmatic default leads us to the conclusion that Psituations each contain a different bear. Suppose I am right in saying that we take the set of trial situations to be the antecedent for $P$ on Scenario Two. Then, to the extent that we still consider (17) to be conversationally appropriate on Scenario Two, it must be that (17) does not presuppose that every P-situation contains a different bear. This suggests that sentences with adverbial quantifiers and indefinites do not in general require a one-to-one mapping from situations to individuals.

18. Von Fintel's approach in particular is an extension of similar ideas in Berman 1987 and Heim 1990.

19. Deriving this presupposition requires among other things a different logical form that includes a new item, von Fintel's topic operator. Throughout, I will just pretend that von Fintel's logical form for (13) is like mine, since I think I can preserve the essence of his account this way.

20. Or more properly the denotation of (13)'s lf.

21 . This assumption is crucial to my argument. Any von Fintelite who objects to it is probably also going to say that, when we evaluate (21) under Scenarios One and Two, we don't use the analogous set to obtain an antecedent for P. So anyone who objects is also going to have to give an explanation for how, in those cases, we can take (21) to make the restricted claim it does without using this set to obtain an antecedent.

22. De Swart 1996 investigates sentences containing adverbial quantifiers and definites. 


\section{References}

Beaver, D. 1995. Presupposition and Assertion in Dynamic Semantics. PhD diss., University of Edinburgh.

Berman, S. 1987. "A Situation-Based Semantics for Adverbs of Quantification." In J. Blevins and A. Vainikka, eds., UMOP 12. GLSA, University of Massachusetts, Amherst.

Buering, D. 1996. "A Weak Theory of Strong Readings." In T. Galloway and J. Spence, eds., Proceedings of SALT VI, 17-34. Ithaca, NY: Cornell University Linguistic Publications.

von Fintel, K. 1994. Restrictions on Quantifier Domains. PhD diss., University of Massachusetts, Amherst.

von Fintel, K. 1996. "A Minimal Theory of Adverbial Quantification." In H. Kamp and B. Partee, eds., Context in the Analysis of Linguistic Meaning. Stuttgart/ Prague: IMS/UFAL.

Heim, I. 1982. The Semantics of Definite and Indefinite Noun Phrases. PhD diss., University of Massachusetts, Amherst.

Heim, I. 1983. "On the Projection Problem for Presuppositions." In M. Barlow, D. Flickinger and M. Westcoat, eds., Proceedings of WCCFL 2, 114-125. Stanford, CA: Stanford Linguistics Association.

Heim, I. 1990. "E-type Pronouns and Donkey Anaphora." Linguistics and Philosophy 13: 137-178.

Heim, I. 1997. Lecture notes on the semantics of tense, MIT.

Heim, I. and A. Kratzer. 1998. Semantics in Generative Grammar. Malden, MA: Blackwell.

Kamp, H. 1981. "A Theory of Truth and Discourse Representation." In J. Groenendijk, T. Janssen and M. Stokhof, eds., Formal Methods in the Study of Language, 277-322. Mathematical Centre, Amsterdam.

Kratzer, A. 1989. "An Investigation of the Lumps of Thought." Linguistics and Philosophy 12: 607-653.

Ogihara, T. 1996. Tense, Attitudes and Scope. Dordrecht: Kluwer.

Percus, O. 1997. Aspects of A. PhD diss., MIT.

Percus, O. 1998. "Some Instructions for the Worldly." To appear in S. Blake, E.-S. Kim and K. Shahin, eds., Proceedings of WCCFL 17, Stanford, CA: CSLI Publications.

Rooth, M. 1992. "A Theory of Focus Interpretation." Natural Language Semantics 1, 75-116.

Schubert, L. and J. Pelletier. 1987. "Problems in the Interpretation of the Logical Form of Generics, Bare Plurals, and Mass Terms." In E. Lepore, ed., New Directions in Semantics, 387-453. London: Academic Press.

Soames, S. 1989. "Presupposition." In D. Gabbay and F. Guenther (eds.), Handbook of Philosophical Logic, Volume IV, 553-616. Dordrecht: Reidel.

de Swart, H. 1996. “(In)definites and Genericity.” In M. Kanazawa, C. Pinon and H. de Swart, eds., Quantifiers, Deduction, and Context. Stanford: CSLI Publications. 